\title{
THE STOCHASTIC FINITE ELEMENT METHOD FOR NUCLEAR APPLICATIONS
}

Conference Paper · June 2016

READS

44

14 authors, including:

\section{Lee Margetts}

The University of Manchester 100 PUBLICATIONS 379 CITATIONS

$$
\text { SEE PROFILE }
$$

A. Luis Cebamanos

The University of Edinburgh

9 PUBLICATIONS 6 CITATIONS

SEE PROFILE
Llion Marc Evans

Culham Centre for Fusion Energy

12 PUBLICATIONS 13 CITATIONS

SEE PROFILE

Paul Mummery

The University of Manchester

126 PUBLICATIONS 1,113 CITATIONS

SEE PROFILE 


\title{
THE STOCHASTIC FINITE ELEMENT METHOD FOR NUCLEAR APPLICATIONS
}

\author{
José David Arregui Mena ${ }^{1}$, Lee Margetts ${ }^{1}$, Llion Evans ${ }^{1,2}$, D. V. Griffiths ${ }^{3}$, Anton \\ Shterenlikht $^{4}$, Luis Cebamanos ${ }^{5}$ and Paul M Mummery ${ }^{2}$ \\ ${ }^{1}$ University of Manchester \\ Oxford Road, Manchester, M13 9PL \\ e-mail: jose.arregui-mena@manchester.ac.uk, lee.margetts@manchester.ac.uk, \\ paul.m.mummery@manchester.ac.uk \\ ${ }^{2}$ Culham Centre for Fusion Energy \\ Culham Science Centre, Abingdon, OX14 3DB \\ e-mail: Llion.Evans@ukaea.uk \\ ${ }^{3}$ Colorado School of Mines \\ 1500 Illinois Street, Golden CO 80401, USA \\ e-mail: d.v.griffiths@mines.edu \\ ${ }^{4}$ University of Bristol, Queen's Building \\ University Walk, Clifton BS8 1TR \\ e-mail:mexas@bristol.ac.uk \\ ${ }^{5}$ University of Edinburgh \\ James Clerk Maxwell Building, Peter Guthrie Tait Road, Edinburgh, EH9 3FD \\ e-mail: 1.cebamanos@epcc.ed.ac.uk
}

Keywords: Random Finite Element Method, Nuclear Energy, Nuclear Fusion.

\begin{abstract}
Nuclear materials are subjected to demanding environments, encountering high temperature gradients and fast neutron fluxes that gradually damage its structure and therefore change the material properties. Some components of a nuclear reactor determine its lifetime, such as the graphite core and steel pressure vessel for fission reactors. In case of fusion reactors the tungsten divertor is expected to be replaced several times during its lifespan. All these materials contain defects and spatial material variability that may contribute to the failure of the component. The Stochastic Finite Element Method or a Random Finite Element Method was chosen in this research to model the spatial material variability in nuclear graphite and other key components of nuclear reactors. This research describes how a direct Monte Carlo Simulation approach was adapted to simulate the calibration of a random field and the modelling of these defects for nuclear graphite. It is also suggested that this methodology can be applied to fusion reactor modelling.
\end{abstract}




\section{INTRODUCTION}

The Stochastic Finite Element Method (SFEM) or Random Finite Element Method (RFEM) is an extension of the FEM that considers the uncertainty of a system that arises through variations in initial conditions, forces, materials or geometry. These uncertainties can be taken into account by representing them through several mathematical tools i.e. random fields [1] or random media techniques [2]. The most common tool to represent a random variable with the SFEM is random fields, which can be described as a series of indexed random variables that describe a stochastic system [1]. A random field can potentially represent the stochastic nature of a system by including a) stochastic forces, b) spatial variability of material properties, or a c) randomly generated geometry (Figure 1). Several branches of the SFEM can be used to include, represent and solve a stochastic model; for a more in detail description of the SFEM the reader is referred to reference [3].

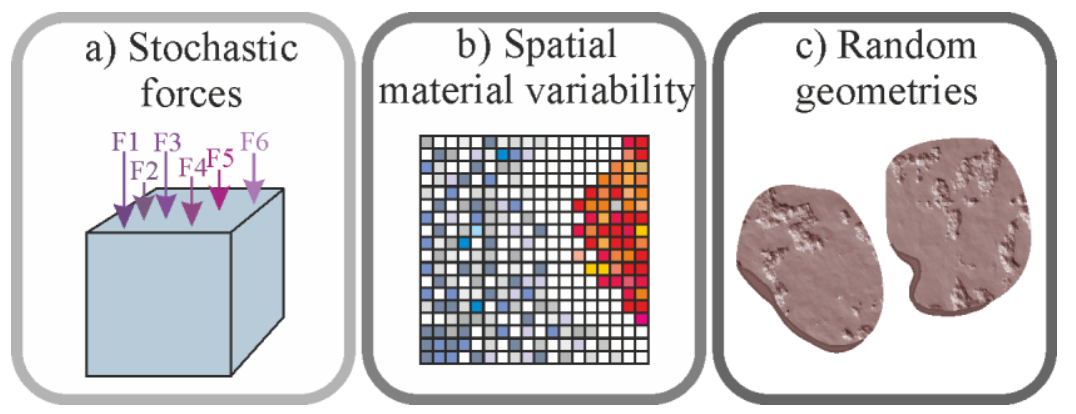

Figure 1. Stochastic variables that can be represented with random fields a) Stochastic forces, b) Spatial material variability, c) Random geometries

Random processes or uncertainties are present up to some degree in most systems. In nuclear reactors several stochastic variables arise from the defects of materials and the environment created by nuclear chain reactions. Figure 2 shows the types of chain reactions occurring in fission and fusion reactors.
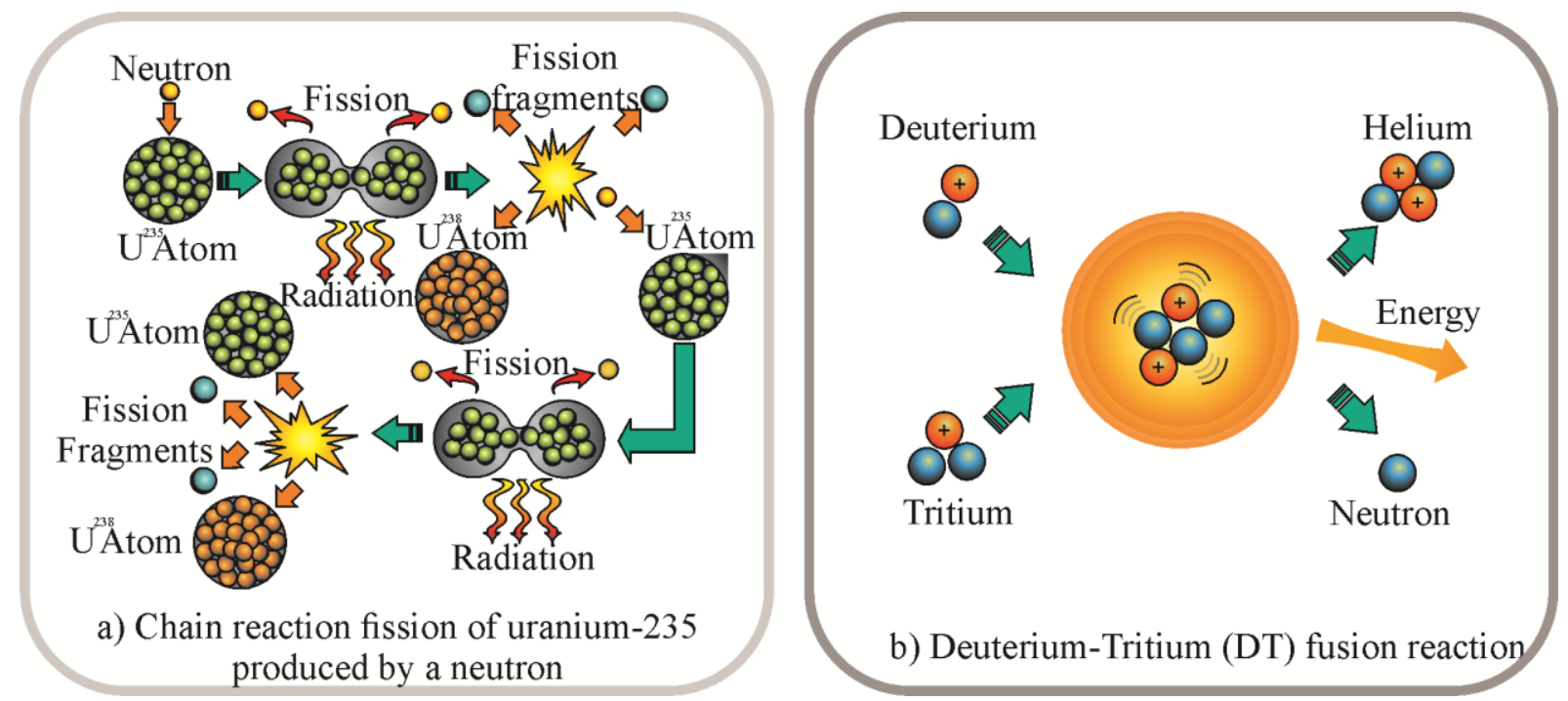

Figure 2. Types of chain reactions in fission and fusion reactors a) Fission reaction of uranium-235 (adapted from [4]), b) Fusion reaction of deuterium and tritium (adapted from [5]) 
The products of fusion and fission chain reactions are fast neutrons, the high energy of these particles produce a progressive damage to the components at the vicinity of these reactions. Furthermore, nuclear reactions can produce a transmutation of an element and generate heat. In this study we propose a methodology to study the effect of these environmental variables combined with the spatial material variability. The first case study proposes a methodology to predict the influence of spatial variability in nuclear graphite for British nuclear reactors. The second case study implements a random field generator of porosity to reproduce similar defects to the ones found in the tungsten components of a fusion reactor.

\section{SFEM FOR FISSION NUCLEAR REACTORS MATERIALS}

A Random Finite Element Method with a direct Monte Carlo Simulation scheme is proposed to study the UK's graphite moderated reactors called Advanced Gas-cooled Reactors (AGRs). The core of these reactors is formed by thousands of graphite bricks that serve as a moderator, a structural support and also provide a repository for fuel rods and other instruments. A summary of the most important parts of an AGR and its core components is shown in Figure 3.

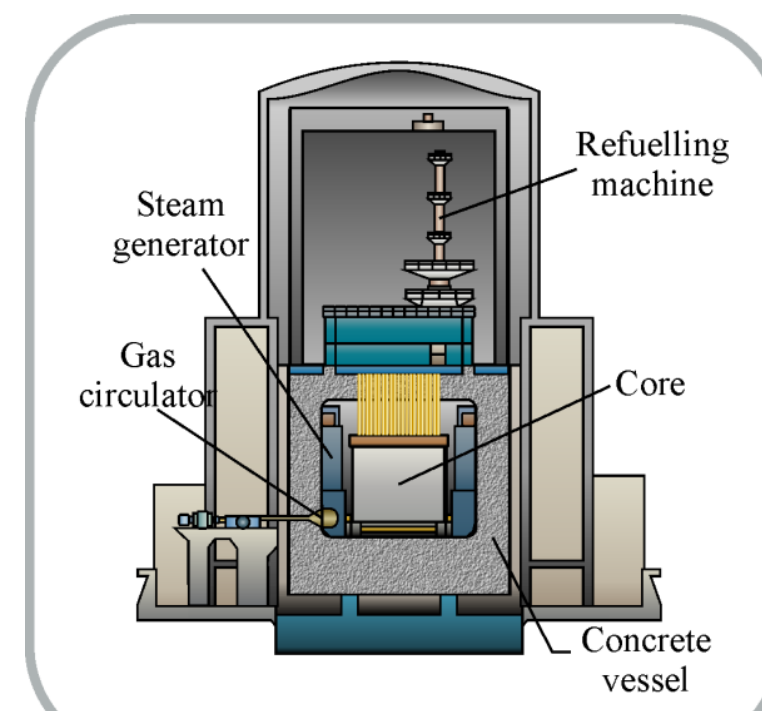

a) Cross section of an AGR

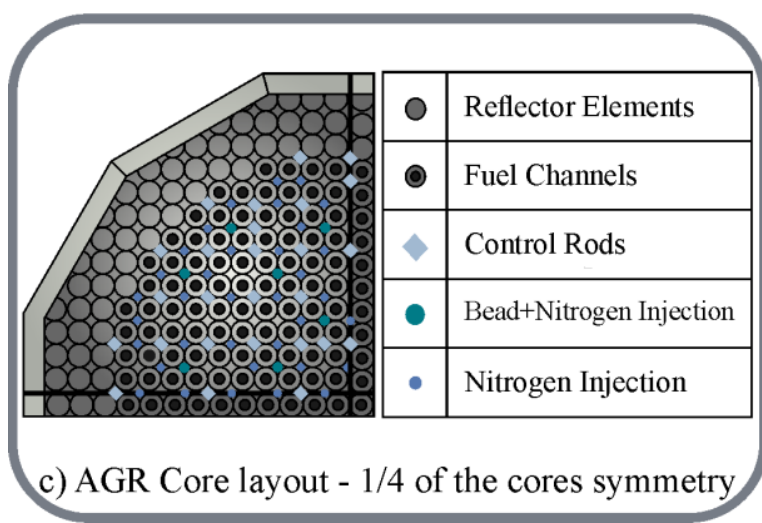

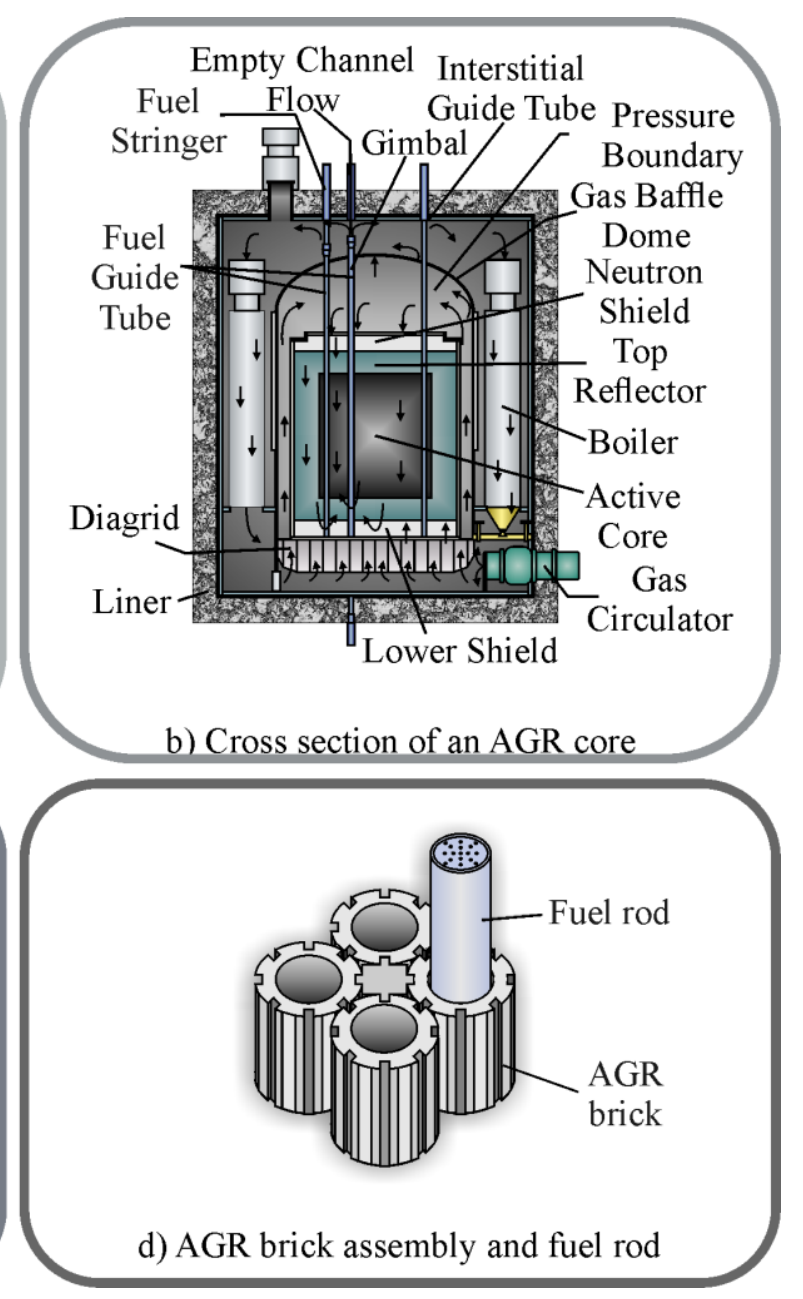

d) AGR brick assembly and fuel rod

Figure 3. Parts of an AGR a) Cross section of an AGR (adapted from [6]), b) Cross section of an AGR core (adapted from [7]), c) AGR core layout (adapted from [8]), d) AGR brick assembly and fuel rod 
The graphite bricks are subjected to several ageing processes that alter the material properties, produce dimensional changes induced by irradiation and temperature gradients and reduce the weight of the components. As a consequence the geometry of the graphite bricks could change. This, in turn, would cause a stress concentration, which may lead to cracking. The total strains $\left(\Delta \varepsilon^{\text {Total }}\right)$ that a graphite brick suffers come from a combination of 4 factors that are: elastic strains $\left(\Delta \varepsilon^{\mathrm{e}}\right)$, creep strains $\left(\Delta \varepsilon^{\mathrm{c}}\right)$, dimensional strains $\left(\Delta \varepsilon^{\mathrm{dc}}\right)$ and thermal strains $\left(\Delta \varepsilon^{\text {th }}\right)$. All these strains are represented in Equation 1.

$$
\Delta \varepsilon^{\text {Total }}=\Delta \varepsilon^{e}+\Delta \varepsilon^{c}+\Delta \varepsilon^{d c}+\Delta \varepsilon^{\text {th }}
$$

Irradiation creep strains are another product of the neutron irradiation, it is considered to relieve high stresses caused by dimensional changes that are produced by irradiation and thermal strains. The deformation of the core and graphite components can potentially impede the normal cooling of the reactor and the loading and unloading of fuel rods. Furthermore, graphite components cannot be replaced in AGRs, this makes them a critical component of AGR lifetime. The complex interactions that damage the graphite core lead to uncertainties in graphite core lifetime predictions and inspections cannot fully determine the actual condition of the components. In order to provide a different approach, the SFEM was adapted to incorporate spatial variability of material properties to the modeling of the thermomechanical response of graphite components.

The hypothesis of this research is that spatial variability in the coefficient of thermal expansion and Young's modulus can have a significant effect on the mechanical performance of nuclear graphite during its in-service lifetime. In order to test this hypothesis, the open source software ParaFEM [9] is being extended to interface with third party software libraries that can generate spatially variable $3 \mathrm{D}$ random fields for any material property.

\subsection{Methodology}

A deterministic and a stochastic approach were used to compare the influence of spatial material variability of material properties. For this comparison a profile of temperature change was applied to the deterministic and stochastic approach. Boundary conditions, temperature profiles are discussed in the next subsections.

In case of the stochastic approach several realizations of random fields were created and then incorporated into a thermoelastic finite element code using a Monte Carlo scheme. At the last step the influence of spatial variability of the material properties on the response variables e.g. strains and stress distributions in nuclear graphite were estimated. To recreate the spatial material variability the local average subdivision method was used. This random field generator requires the mean values, standard deviation and correlation length as inputs. An in detail explanation of the local average subdivision method can be found in reference [10]. The realisations produced with this random field generator were used as an input in the finite element software ParaFEM. For the deterministic approach only one simulation was created with an average of the material properties listed in Subsection 2.2. Both methodologies are summarised in Figure 4. 


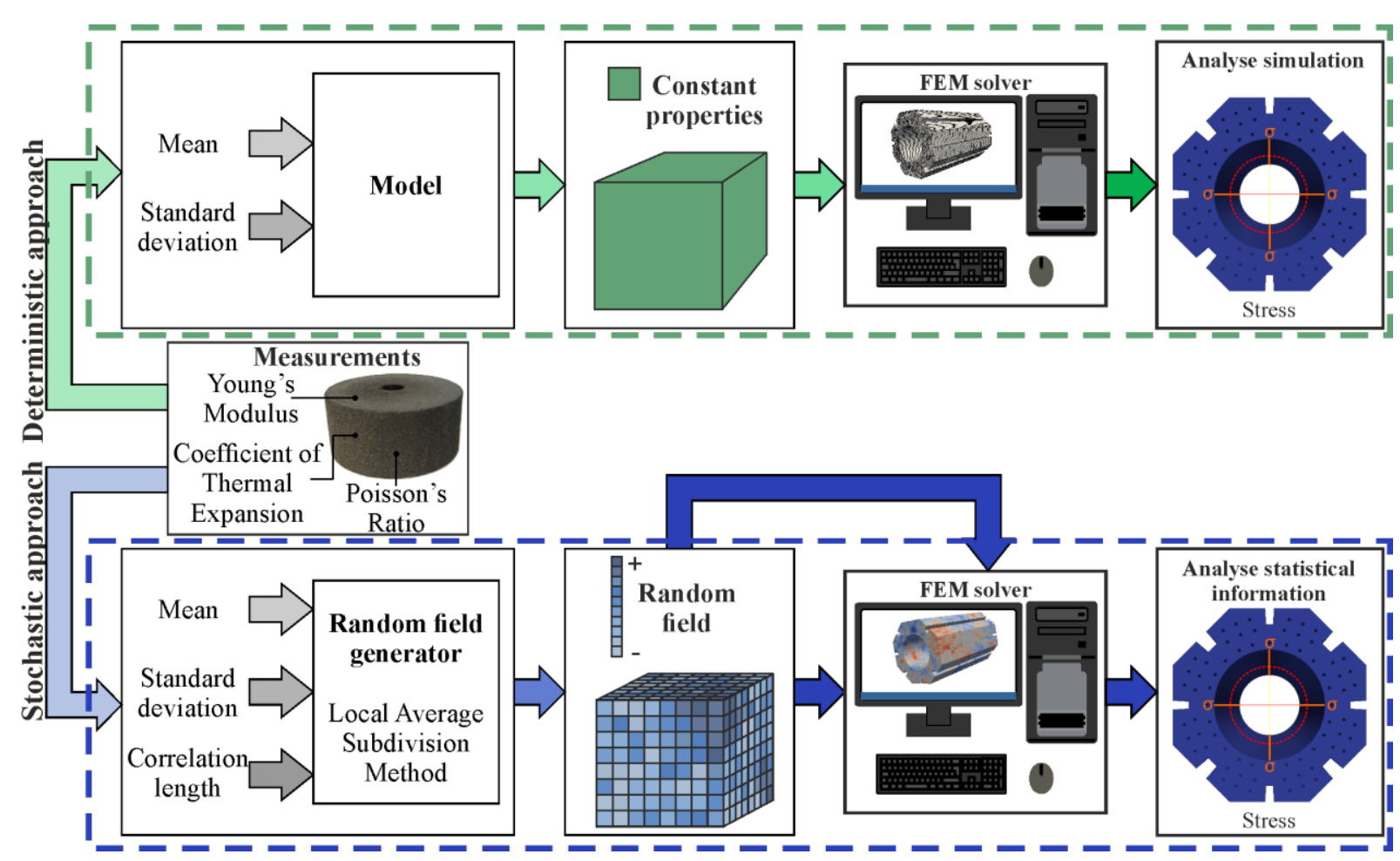

Figure 4. Methodologies for the deterministic and stochastic approach

\subsection{Material properties}

The material properties chosen for these realisations were obtained from [11] (Table 1); the material properties correspond to the average values for isotropic graphite. The standard deviation for the coefficient of thermal expansion was estimated by the authors. The correlation length was also defined by the authors due to the lack of calculations for this parameter. The correlation length is an important parameter required to describe the fluctuation of material properties in a random field. This value is a given length where the values of the random fields are correlated, beyond this distance the correlation between two data points tend to zero.

\begin{tabular}{lll}
\hline $\begin{array}{l}\text { Material properties for } \\
\text { virgin isotropic graphite }\end{array}$ & Mean values & Standard deviation \\
\hline $\begin{array}{l}\text { Mean coefficient of } \\
\text { thermal expansion }\end{array}$ & $\begin{array}{l}4.35 \times 10^{-6} \\
\left(\mathrm{~mm} / \mathrm{mm}^{\circ} \mathrm{C}\right) \underline{[11]}\end{array}$ & $\begin{array}{l}4.35 \times 10^{-7} \\
\left(\mathrm{~mm} / \mathrm{mm}^{\circ} \mathrm{C}\right)\end{array}$ \\
$\begin{array}{l}\text { Poisson's ratio } \\
\text { Dynamic Young's }\end{array}$ & $0.2[11]$ & \\
$\begin{array}{l}\text { Modulus } \\
\begin{array}{l}\text { Correlation length } \\
\text { in all directions }\end{array}\end{array}$ & $10 \mathrm{GPa} \underline{[11]}$ & \\
\hline
\end{tabular}

Table 1: Material properties for all the analysis.

A relationship between Young's modulus and Coefficient of Thermal Expansion (CTE) was used. This relationship is given by equation (2)

$\mathrm{E}(\operatorname{random})=\alpha($ random $) /$ constant 
where E is Young's Modulus, $\alpha$ is the CTE and a constant. Therefore each Young's modulus value for an element would be calculated from the CTE random field value using equation (2).

\subsection{Boundary conditions and field variables}

The boundary conditions used for this research were a modified version of the 3-2-1 rule. The objectives of these boundary conditions are to allow the free thermal expansion, to minimize the stress concentrations around the fixed nodes and to remove any rigid body motions. Figure 5 shows the chosen boundary conditions selected for all the simulations.

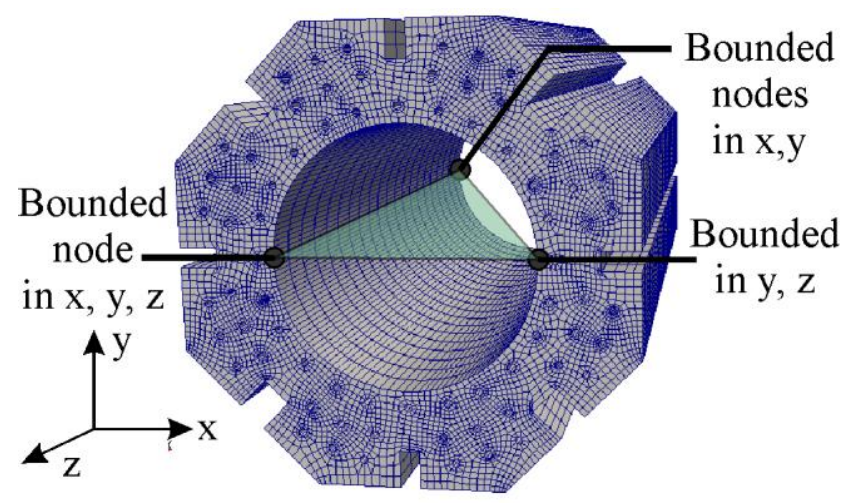

Figure 5. Boundary conditions used for this study

A similar temperature change profile as the ones found in AGR brick was applied to produce thermal expansion. Figure 6 shows this temperature change profile applied to the simulations.

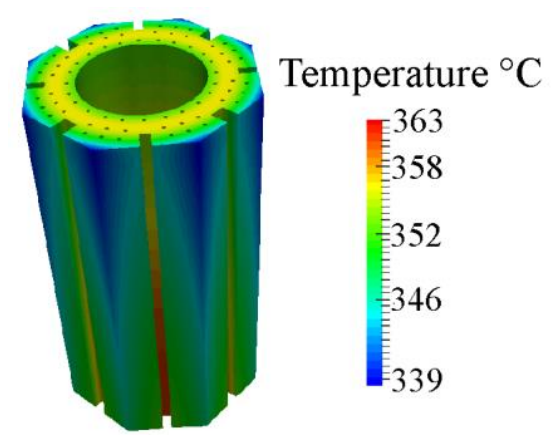

Figure 6. Temperature change profile for all the simulations

\subsection{Results and discussion}

The results of the deterministic and the stochastic approaches are compared in Figure 7. As can be seen on Figure 7 the deterministic approach produces stresses orders of magnitude less than by stochastic modelling. In the model where the stochastic spatial material variability was considered the stresses can reach a magnitude of $4.5 \mathrm{MPa}$. It is important to note that these stress concentrations would not lead to the cracking of graphite bricks. However, the combination of material spatial variability with thermal expansion, irradiation induced dimensional change and creep strains can increase these stress concentrations that might lead to the cracking of a brick. 


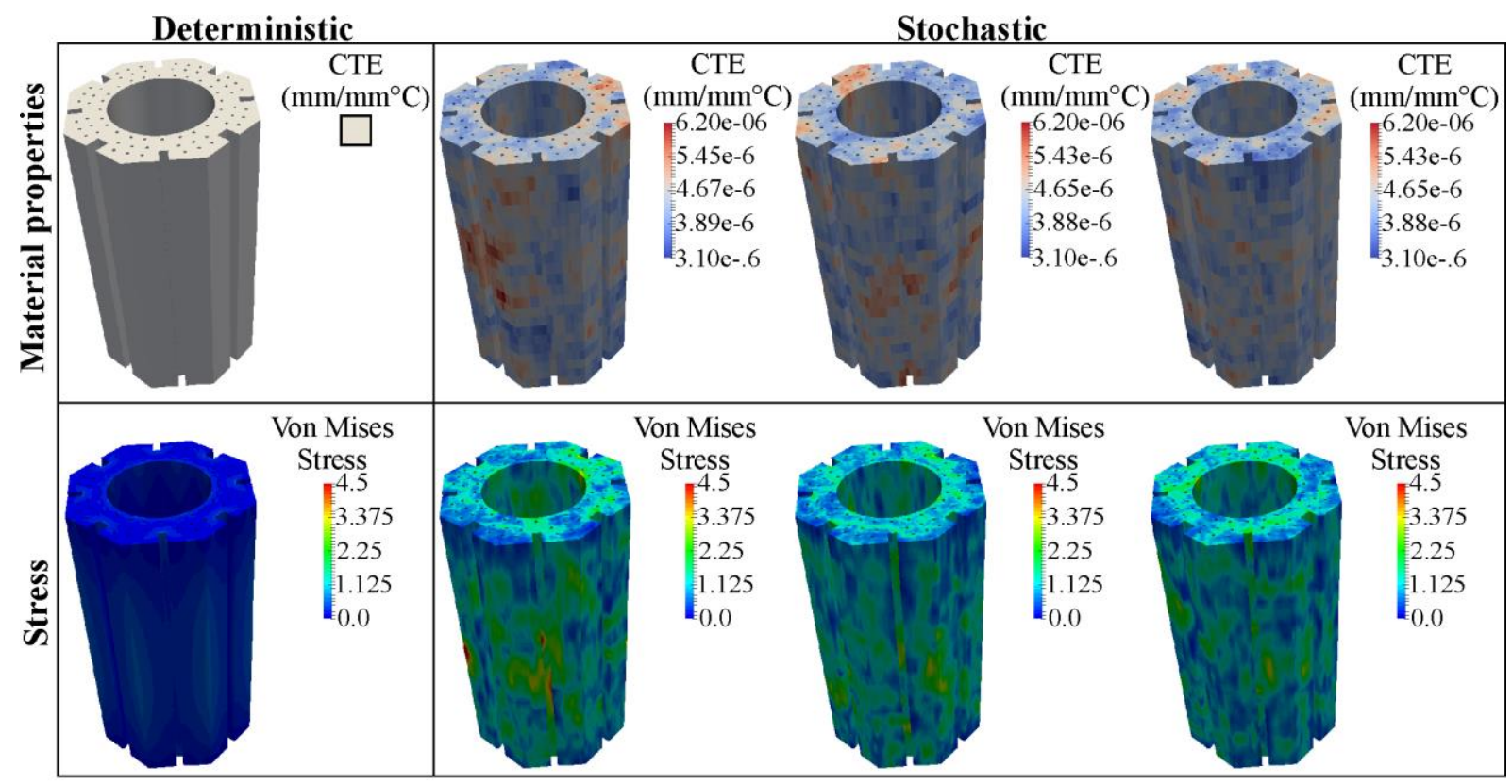

Figure 7. Von Misses stress comparison between the deterministic and stochastic approach

\subsection{Conclusions}

Spatial material variability is not usually considered for the modelling of nuclear graphite components. Variability of mechanical properties may be one of the factors that lead to the early cracking of nuclear components. The results show how material spatial variability increases the magnitude of Von Mises stress. Furthermore, stress concentrations are found in regions where they are not expected.

The current simulations only use only the estimations to recreate the input parameters for the correlation length. A new study focuses on calibrating the random fields from density and Young's modulus measurements made in a billet (Figure 8). These measurements will serve to obtain the average, standard deviation and correlation length for future simulations.

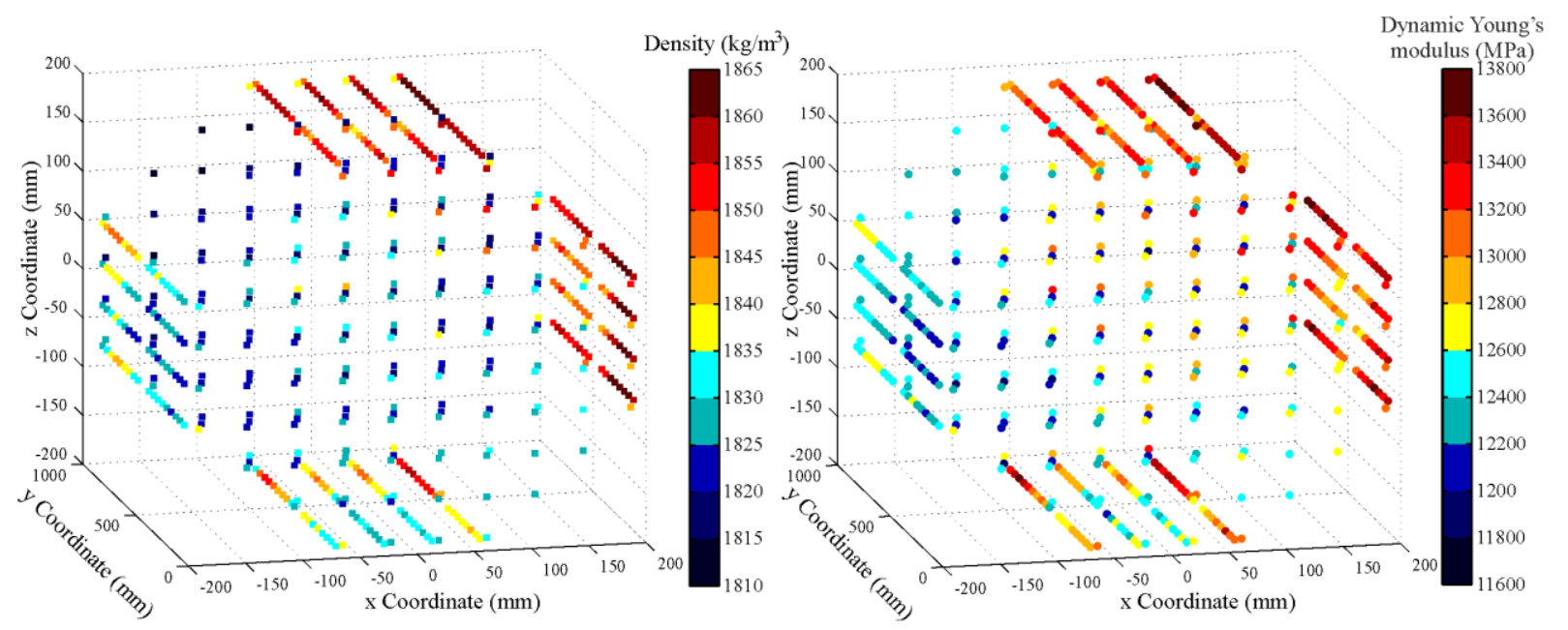

Figure 8. Density and dynamic Young's modulus measured in a graphite billet 
Further improvements to the simulation of graphite modelling are to include a multiscale model by combining cellular automata modelling and the Finite Element Method (FEM) [12]. The aforementioned cellular automata methodology requires information of the crystal orientation of the material; this data has been obtained for nuclear graphite [13]. A random field generator can be modified to use these to implement the cellular automata methodology.

\section{SFEM FOR FUSION NUCLEAR REACTORS MATERIALS}

Materials in a fusion nuclear reactor need to endure extreme conditions. High thermal loads, high neutron fluxes and plasma erosion produce changes and damage in the materials that surround the reactions [14]. Two different designs lead the research to create future fusion reactors: magnetic confinement and inertial confinement. The magnetic confinement approach is currently considered to be the most technologically developed concept of the two. Numerous relatively small tokomaks already exist, but it is known that due to scaling laws that energy output increases at a greater rate than the energy input as plasma volume increases. For this reason, a much larger tokomak is being built in Cadarache in France called ITER [15]. Figure 9 shows the design and interior cross section of a tokamak. The objective of this reactor is to demonstrate the scientific feasibility of sustaining fusion reactions at electricity generating scales and producing an output ten times that of the energy required to initiate the process [16].

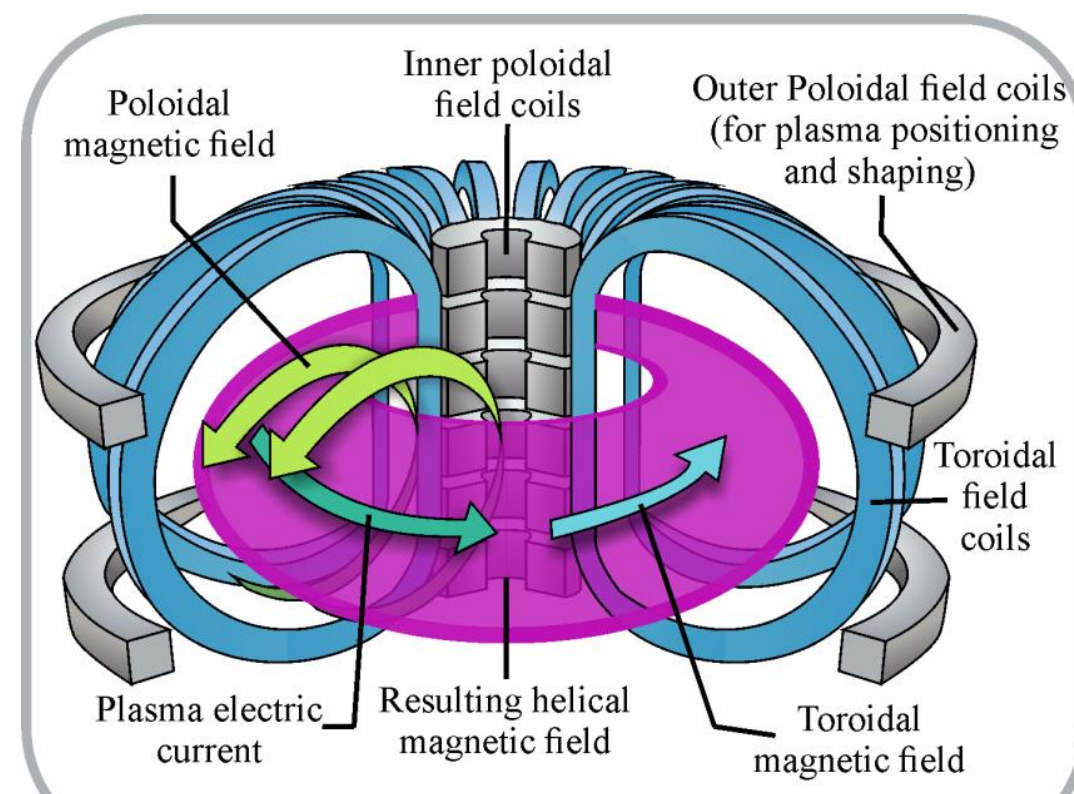

a) Schematic Tokamak design

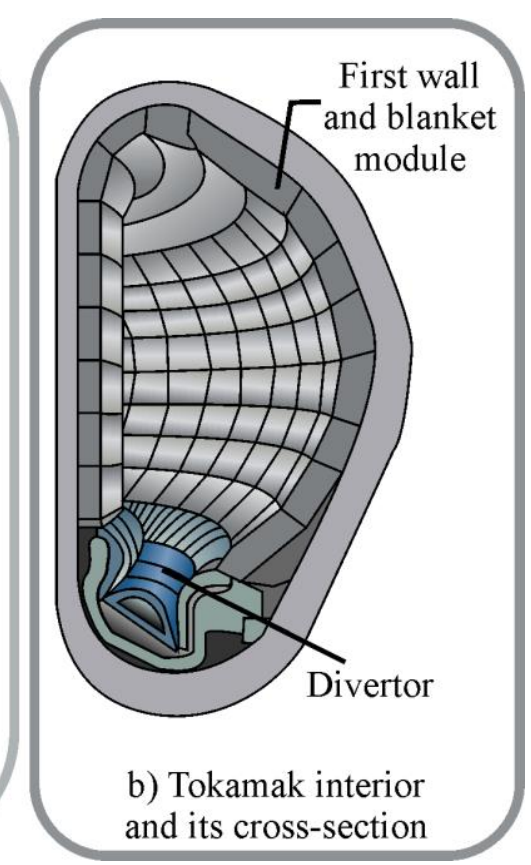

and its cross-section

Figure 9. Tokamak system, a) Schematic of a Tokamak design (adapted from [17]), b) Tokamak interior and its cross-section (adapted from [18])

Vacuum plasma-sprayed tungsten (VPS-W) is a candidate for coating the walls of future fusion components [19]. The VPS-W is a complex material that contains a random porous structure; this porosity strongly influences the thermomechanical behavior of this material [20]. A previous study by Zivelonghi [21] combines synchrotron tomography and ImageBased FEM to model the thermomechanical response of VPS-W. Image-Based FEM uses stacks of $2 \mathrm{D}$ slices of a material to recreate $3 \mathrm{D}$ models of the intricate structures. ImageBased FEM have also been implemented to study other fusion materials such as carbon fiber composites [22]. However, Image-Based FEM is limited to the size, resolution and capacity 
of the tomography equipment, thus large samples with highly detailed porous structure cannot be model through this technique. The information of small geometrical models can be used to calibrate a random field that mimics the porous structure found in VPS-W. These random fields can be incorporated to a SFEM model to calculate the response of larger components covered with VPS-W.

The next subsections describe the random field generator capable of recreating porous structures and some preliminary results.

\subsection{Porosity random field generator}

A random field generator proposed by Paiboon et al. [23] is capable of producing random fields that represent porosity in materials. This random field generator allows the user to control the volume fraction of porosity and pore size by controlling the correlation length and percentage of porosity. Examples of some realisations are shown in Figure 10.
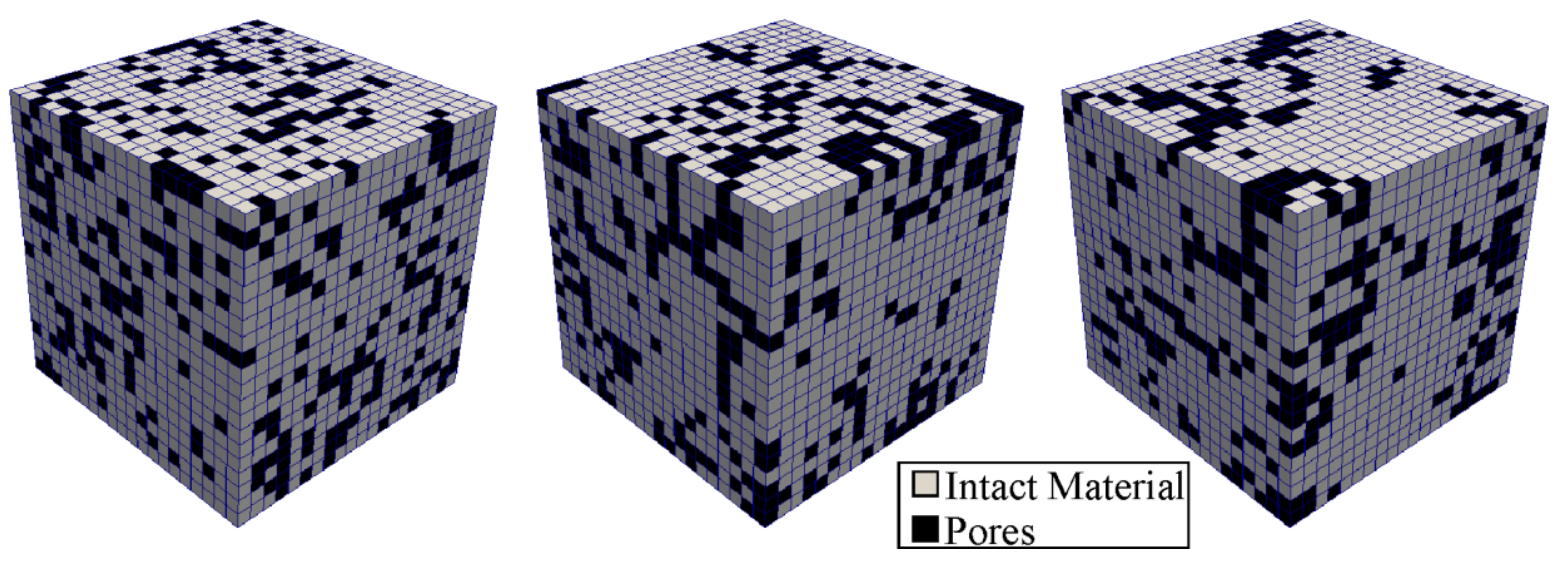

Figure 10. Realisations of porous materials

This random field generator is a modified version of the Local Average Subdivision method [10]. In this random field the intact material is assigned with a given material property value and the porous material property value are 100 times smaller than the value for intact material.

Several types of software are available to create 3D models from computer tomography data. The geometrical information of the pore can be manipulated and used to obtain the average size of the pore structure. Once the mean size of the pores has been found, it can be used as an input file for the aforementioned random field generator. The final step would be to assign the random field material property values to model the thermomechanical properties of larger components.

To test the influence of porosity in tungsten some preliminary results were obtained by calculating the effective material properties of one realisation. 


\subsection{Material properties}

The material properties used for the intact material in the simulations are given in Table 2.

\begin{tabular}{ll}
\hline $\begin{array}{l}\text { Material properties for } \\
\text { virgin isotropic graphite }\end{array}$ & Values \\
\hline $\begin{array}{l}\text { Poisson's ratio } \\
\text { Young's Modulus }\end{array}$ & 0.28 \\
$\begin{array}{l}\text { Correlation length } \\
\text { in all directions }\end{array}$ & $0.1 \mathrm{GPa}$ \\
$\begin{array}{l}\text { Target porosity for } \\
\text { porous simulation }\end{array}$ & $0.8 \%$ \\
\hline
\end{tabular}

Table 2: Material properties for intact tungsten material.

\subsection{Boundary conditions and field variables}

The finite element mesh is formed by a cubic block of materials of $1000 \mathrm{~mm}$ side with $100 \times 100 \times 1008$ node cubic elements. The chosen boundary conditions are known as "tied freedom", with these boundary conditions the cube is forced to be deformed in an ideal cube, Figure 27 shows the geometry and boundary conditions. From this deformation the calculation of the elastic properties can be easily calculated from the elastic theory. The boundary conditions are: the base of the cube can move in $\mathrm{x}$ and $\mathrm{y}$ direction, the back left can move in the $y-z$ plane, the right face in only allowed to movie in the $z-x$ plane. The freedoms of the top face are tied in the $\mathrm{z}$ direction, the front left face freedoms are restrained in the in the $\mathrm{y}$ direction, finally the $x$ freedoms are tied in the right front face. A vertical force is applied at the top of the cube with a value of $\mathrm{Q}=\mathrm{L} \times \mathrm{L}$.

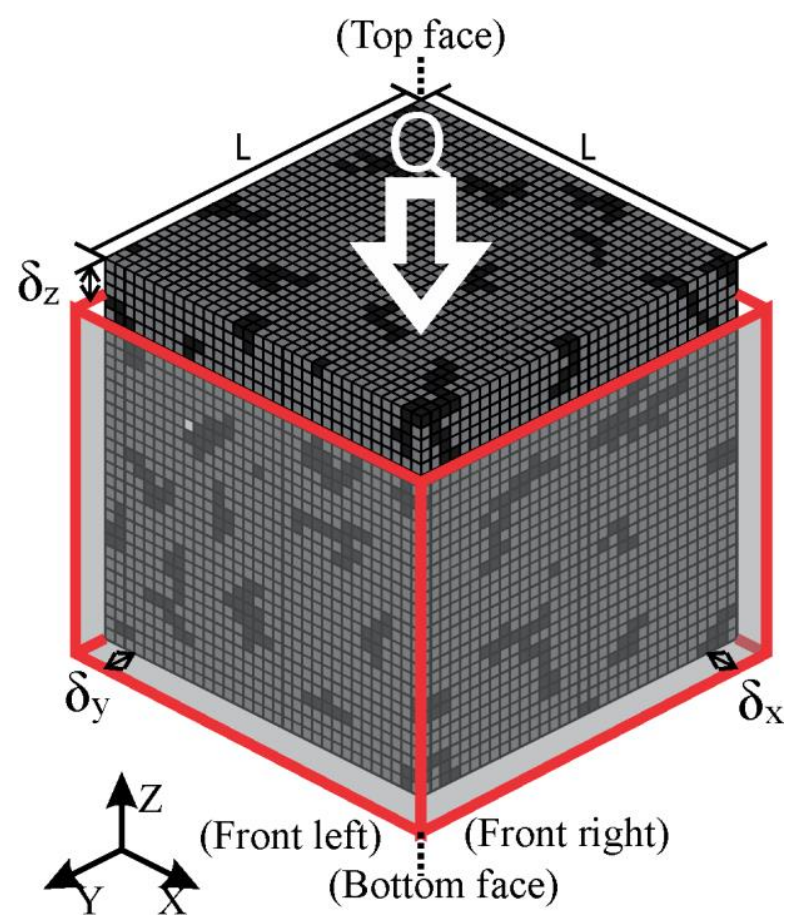

Figure 11. Compression test of a porous material to determine the influence of porosity in graphite 
In order to obtain the effective properties of graphite the values for the deformations in each direction $\delta_{\mathrm{x}}, \delta_{\mathrm{y}}$ and $\delta_{\mathrm{z}}$ are retrieved from each simulation and substituted in the following equations:

$$
\begin{aligned}
& E=\frac{Q}{L \delta_{z}} \\
& v_{x}=\frac{\delta_{x}}{\delta_{z}} \\
& v_{y}=\frac{\delta_{y}}{\delta_{z}}
\end{aligned}
$$

where $\mathrm{E}$ is the effective elastic Young's modulus, $\mathrm{Q}$ is the loading force at the top of the cube and $v_{\mathrm{x}}$ and $v_{\mathrm{y}}$ are the effective Poisson's ratios in $\mathrm{x}$ and $\mathrm{y}$ direction.

\subsection{Results and discussion}

The results of the effective material properties of the deterministic and the stochastic simulations are summarised in Figure 12.
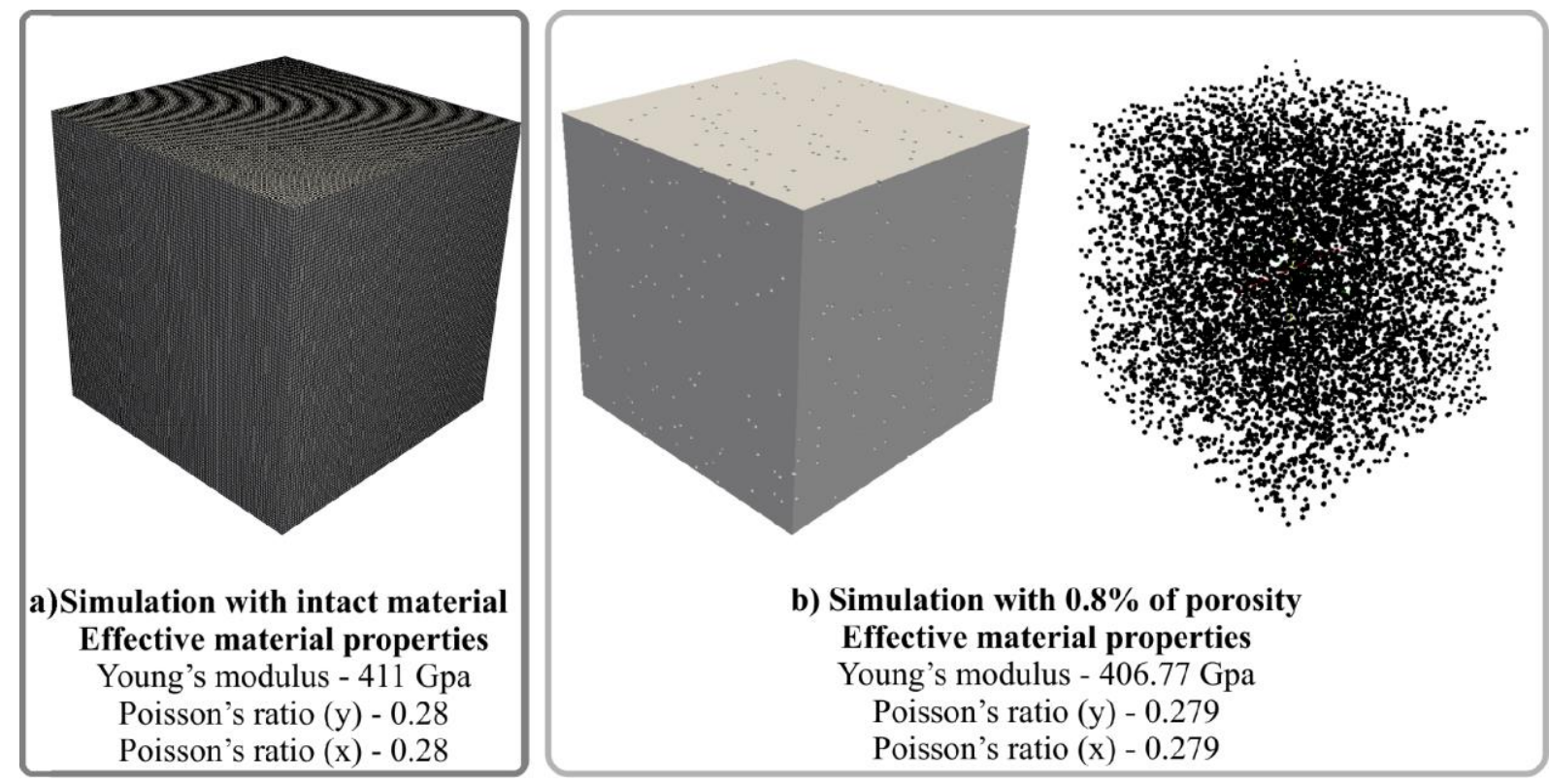

Figure 12. Results a) A deterministic simulation with intact material, b) Intact material and porous structure of the simulation

These tests show how porosity can affect the effective mechanical properties of VPS-W. The effective Young's modulus and Poisson's ratio remain fairly unaffected by this porous volume fraction. However, the extreme thermal loads and neutron dose would cause a more significant change on the mechanical behaviour of VPS-W. Other volume fractions will be used in future research to test different scenarios.

\subsection{Conclusions}

A random field to simulate the porous structure of VPS-W is presented in this study. A methodology to understand the effective Young's modulus and Poisson's ratio is proposed. 
The software ParaFEM will be modified to analyse the thermomechanical response of different volume fractions of porosity in VPS-W using this random field generator.

\section{GENERAL CONCLUSIONS}

- This paper has found that the SFEM has the potential of simulating spatial material variability for nuclear materials. The simulated material properties for graphite, for example, Young's modulus and strength can be calibrated to experimentally measured values. This finding provides confidence for future work concerning the prediction of graphite behaviour over its lifetime in particular, the effect of microcracking of the bulk material. A criterion for crack initiation has to be developed and further work is required to test the accuracy of this method

- A random field for porous materials has been proposed to analyse the thermomechanical response of large components covered with VPS-W. The SFEM would enable to measure the thermomechanical response of whole parts of a fusion reactor. Moreover, preliminary results to obtain the effective material properties of VPS-W are provided.

\section{REFERENCES}

[1] E. Vanmarcke, Random Fields: Analysis and Synthesis. World Scientific, 2010

[2] S. Torquato, Random Heterogeneous Materials: Microstructure and Macroscopic Properties. Springer New York, 2005

[3] J. D. Arregui-Mena, L. Margetts, P. M. Mummery, The Practical Stochastic Finite Element Method, Arch. Comput. Methods Eng. (2014)

[4] R. A. Knief, Nuclear Engineering: theory and technology of commercial nuclear power. Second Edition, 2008

[5] "ITER - the way to new energy" [Online]. Available: http://www.iter.org/sci/whatisfusion. [Accessed 29 August 2013]

[6] D. G. Cacuci, Handbook of nuclear engineering. New York, Springer, 2010

[7] A. G. Steer, AGR core design, operation and safety functions. Management of ageing in graphite reactor core, 2007

[8] E. Nonbol, Description of the Advanced Gas Cooled Type of Reactor (AGR). Reactors in Nordic Surroundings. Roskilde, Denmark: Ris $\propto$ National Laboratory

[9] I. M. Smith, D. V. Griffiths, L. Margetts, Programming the Finite Element Method, Fifth Edition. Wiley, United Kingdom, 2014

[10] G. A. Fenton, D.V. Griffiths, Risk Assessment in Geotechnical Engineering. John Wiley \& Sons, 2008

[11] D. K. L. Tsang, B. J. Marsden, The development of a stress analysis code for nuclear graphite components in gas-cooled reactors. Journal of Nuclear Material, Vol 350, 2006.

[12] A. Shterenlikht, L. Margetts, Three-dimensional cellular automata modelling of cleavage propagation across crystal boundaries in polycrystalline microstructures. Proceedings of the Royal Society A: Mathematical, Physical and Engineering Sciences, Vol 471 
[13] M. Haverty, The microstructure, texture and thermal expansion of nuclear graphite. Thesis, Manchester, UK, The University of Manchester, 2015

[14] L1.M. Evans, Thermal Finite Element Analysis of Ceramic/Metal joining for fusion using X-ray tomography data. Thesis, UK, The University of Manchester, 2013

[15] International Atomic Agency, Fusion Physics. Vienna, Austria, 2012

[16] “The tokamak" [Online], Available: http://www.ccfe.ac.uk/tokamak.aspx, [Accessed 01 March 2016]

[17] [Online]. Available: http://www.jet.efda.org/wp-content/gallery/graphics/jg05-5371c.jpg. [Accessed 29 August 2013].

[18] "Specific manufacturing : heat removal system with cooling water system," [Online]. Available: http://www.atmostat-alcen.com/en/activities/specific-manufacturing. [Accessed 29 August 2013].

[19] Greuner, H. Bolt, B. Böswirth, S. Lindig, W. Kühnlein, T. Huber, K. Sato, S. Suzuki, Vacuum plasma-sprayed tungsten on EUROFER and 316L: Results of characterisation and thermal loading tests. Fusion Engineering and Design, 2005

[20] Exploring the pores in a heavy metal: the 3D microstructure of coatings for nuclear fusion reactors is revealed by high-resolution synchrotron microtomography [Online], http://www.esrf.eu/news/spotlight/spotlight113, [Accessed 01 March 2016]

[21] A. Zivelonghi, Thermomechanical behavior of two heterogeneous tungsten materials via 2D and 3D Image-Based FEM. Thesis, Technischen Universität München, München,Germany, 2010

[22] L1. M. Evans, L. Margetts, V. Casalegno, L. M. Lever, J. Bushell, T, Lowe, A. Wallwork, P. Young, A. Lindemann, M. Schmidt, P.M. Mummery, Transient thermal finite element analysis of CFC-Cu ITER monoblock using X-ray tomography data, Fusion Engineering and Design. Vol 100, 2015

[23] J. Paiboon, D.V. Griffiths, Jinsong Huang, G. A. Fenton, Numerical analysis of effective elastic properties of geomaterials containing voids using 3D random fields and finite elements. International Journal of Solids and Structures, Vol 50, 2013 